\title{
ROTAMAK CONFINEMENT-POWER-CURRENT RELATIONSHIPS AND R.F. LOADING RESISTANCE
}

\author{
P. M. Bellan \\ California Institute of Technology, Pasadena, CA 91125, U.S.A. \\ (Received 19 April 1988 ; and in revised form 6 December 1988)
}

\begin{abstract}
The relationships between input power, driven current, energy confinement, temperature and density are examined in detail for existing rotamak experiments. Additionally, the loading resistance presented to the r.f. power supply and the density at which this resistance peaks are calculated (for typical experiments this density corresponds to an optimum operating point since maximum power is coupled to the plasma).
\end{abstract}

\section{INTRODUCTION}

A STEADY-STATE toroidal current could be used to both confine and heat the plasma in a steady-state toroidal fusion device. Steady-state toroidal current drive has been amply demonstrated in the rotamak, a novel toroidal device (Hugrass et al., 1980; DuRANCE et al., 1982, 1987; JoNes, 1986; Collins et al., 1988; KNIGHT, 1988). However, the confinement properties of the rotamak have been modest compared to other devices. Furthermore, each experiment had an empirically determined optimum operating pressure at which maximum plasma current was obtained. The purpose of this paper is to show how these features and several other experimentally observed operating points of the rotamak can be deduced with reasonable accuracy from fundamental equations.

DoNNelly et al. (1987) studied the global power balance relations for rotamaks rotating synchronously with the applied rotating magnetic field and considered several energy loss mechanisms each of which would dominate in a particular temperature regime. In this paper we, in contrast, will discuss power balance relations for rotamaks that are not necessarily rotating synchronously with the applied field and also will propose that the results of experiments to date (all low temperature) can be explained on the basis of energy loss occurring within an order of magnitude of the ion acoustic velocity. The predictions of this paper will be compared in detail to the experimental data of two particularly well diagnosed rotamak experiments: the $17 \mathrm{~kW}$ input power, prolate case of DURANCE et al. (1987) at the Australian Nuclear Science and Technology Organization and the $8 \mathrm{mT}$ fill case of KNIGHT (1988) at Flinders University. For clarity and to avoid excessive repetition in the text, relevant parameters of these two experiments are listed in Table 1. Parameters marked with an asterisk were not explicitly quoted in the original references, but may be easily inferred from the quoted parameters.

\section{PRESSURE-POWER-CURRENT RELATIONSHIPS}

One of the fundamental parameters of a plasma device is the energy confinement time $\tau_{E}$ defined by

$$
\tau_{E}=\frac{3}{2 P} \int \mathrm{d}^{3} r \mathscr{P}
$$


TABLE 1. EXPERIMENTALLY MEASURED PARAMETERS

\begin{tabular}{llll}
\hline Parameter & Units & Durance et al. & Knight \\
\hline Time into shot & $\mathrm{ms}$ & 14 & 19 \\
$\omega$ & radians $\mathrm{s}^{-1}$ & $6.3 \times 10^{6}$ & $6.3 \times 10^{6}$ \\
$\widetilde{B}$ & $\mathrm{G}$ & 17 & 15 \\
$\mu$ & a.m.u. & 1 & 40 \\
$n_{\max }$ & $\mathrm{m}^{-3}$ & $7.5 \times 10^{18}$ & $1.2 \times 10^{19}$ \\
$T_{\text {emax }}$ & $\mathrm{eV}$ & 10 & 6.5 \\
$\mathscr{P}_{\max }$ & $\mathrm{Pa}$ & 12.5 & 12 \\
$Z$ & & 1 & 2.3 \\
$I$ & $\mathrm{kA}$ & 1.3 & 2.7 \\
$P$ & $\mathrm{~kW}$ & 17 & $22 \pm 8$ \\
$\tau_{E}$ & $\mu \mathrm{s}$ & 5 & $-1.04^{*}$ \\
$r_{0}$ & $\mathrm{~cm}$ & $8^{*}$ & 4.2 \\
$R_{\text {sep }}=\sqrt{2} r_{0}$ & $\mathrm{~cm}$ & 11 & 0.2 \\
$\alpha$ & & 0.8 & $0.75^{*}$ \\
$\varepsilon$ & & $0.3^{*}$ & $10.9^{*}$ \\
$\ln \Lambda$ & & $11.6^{*}$ & $5.3 \times 10^{7 *}$ \\
$\nu_{\text {ei_- }}$ & $\mathrm{s}^{-1 *}$ & $8 \times 10^{6}$ & $2^{*}$ \\
$a$ & $\mathrm{~cm}$ & 1.8 & $1^{*}$ \\
$R_{\text {load }}$ & $\mathrm{Ohms}$ & & \\
\hline
\end{tabular}

where $P$ is the input power, $\mathscr{P}$ is the electron pressure, and the integral is over the volume of the plasma (for simplicity we will ignore the ion energy content which is considerably smaller than the electron energy content for the rotamak devices discussed here).

KNIGHT (1988) showed that the pressure profiles of a rotamak experiment at Flinders University were described reasonably accurately by the SoLov'Ev (1976) solution to the Grad-Shafranov equation. While this solution might be somewhat less accurate for other experiments, it has the enormous advantage of being analytically tractable. Hence, in this paper we will also use the Solov'ev solution. Since the analysis that follows depends on integrals over profiles, the results will not depend strongly on the detailed shape of the profiles and so the Solov'ev model should be quite accurate even if does not precisely describe the detailed profile.

The Grad-Shafranov equation assumes toroidal symmetry so that the magnetic field is given by

$$
\mathbf{B}=\frac{1}{2 \pi} \nabla \psi \times \nabla \theta+B_{\theta} r \nabla \theta
$$

where $\psi$ is the poloidal flux, i.e. $\psi=\int B_{2} 2 \pi r \mathrm{~d} r$. The Grad-Shafranov equation [obtained by substituting (2) into the MHD pressure balance relation $\nabla \mathscr{P}=\mathbf{J} \times \mathbf{B}$ ] is

$$
r \frac{\partial}{\partial r} \frac{1}{r} \frac{\partial \psi}{\partial r}+\frac{\partial^{2} \psi}{\partial z^{2}}+(2 \pi)^{2}\left[\mu_{0} r^{2} \frac{\partial \mathscr{P}}{\partial \psi}+B_{\theta} r \frac{\partial\left(B_{\theta} r\right)}{\partial \psi}\right]=0 .
$$

From (2)-(3) it is easily seen that the toroidal current density is

$$
J_{\theta}=2 \pi r \frac{\partial \mathscr{P}}{\partial \psi}+\frac{2 \pi}{\mu_{0}} \frac{\partial\left(B_{\theta} r\right)}{\partial \psi} B_{\theta}
$$


For the rotamaks under consideration $B_{\theta}=0$ so the last term (force-free current) in (4) vanishes (it of course will also vanish if $B_{\theta} \sim r^{-1}$ ).

The Solov'ev model assumes that the pressure varies linearly with $\psi$ and that

$$
\psi=\psi_{0} \frac{r^{2}}{r_{0}^{4}}\left(2 r_{0}^{2}-r^{2}-\alpha^{2} z^{2}\right)
$$

where $r_{0}$ is the radius of the magnetic axis, $\psi_{0}$ is the flux on the magnetic axis $r=r_{0}$, $z=0$, and $\psi$ vanishes on an elliptical separatrix. $\alpha$ is the ellipticity factor of this separatrix; the maximum radial dimension of the separatrix is $R_{\mathrm{sep}}=\sqrt{2} r_{0}$ and the maximum $z$ dimension is $R_{\text {sep }} / \alpha$. The separatrix is assumed to be approximately coincident with the wall. Inserting (2) and (5) in (3) gives

$$
\frac{\partial \mathscr{P}}{\partial \psi}=\frac{2 \psi_{0}}{\pi^{2} \mu_{0} r_{0}^{4}}\left(1+\alpha^{2} / 4\right) .
$$

If we define $\varepsilon$ as the ratio of the pressure on the separatrix to the pressure on the magnetic axis $\mathscr{P}_{\max }$, then the pressure can be written as

$$
\mathscr{P}=\left[\varepsilon+(1-\varepsilon) \frac{\psi}{\psi_{0}}\right] \mathscr{P}_{\max }
$$

which with (6) gives

$$
\psi_{0}=-\pi r_{0}^{2}\left[\frac{\mu_{0} \mathscr{P}_{\max }(1-\varepsilon)}{2\left(1+\alpha^{2} / 4\right)}\right]^{1 / 2}
$$

(note that we have chosen the negative root so as to make the sign of $\psi_{0}$ consistent with a DC toroidal current produced by electrons traveling in the positive $\theta$ direction).

The corresponding current profile is

$$
J_{\theta}=-\frac{2 r}{r_{0}^{2}}\left[\frac{2\left(1+\alpha^{2} / 4\right) \mathscr{P}_{\max }(1-\varepsilon)}{\mu_{0}}\right]^{1 / 2} .
$$

If $\varepsilon \approx 1$, the plasma is essentially unconfined: there is uniform pressure right up to the wall, and the wall (rather than the $\mathbf{J} \times \mathbf{B}$ force) supports the plasma pressure. In contrast, if $\varepsilon \approx 0$ then the plasma is entirely confined by the magnetic field and exerts no pressure on the wall.

We can use (6) and (7) to evaluate the integral in (1) to obtain 


$$
\tau_{E}=\frac{3}{2 P} \int_{-R_{\mathrm{sep}} / \alpha}^{R_{\mathrm{sep}} / \alpha} \mathrm{d} z \int_{0}^{\left(R_{\mathrm{sep}}^{2}-\alpha^{2} z^{2}\right)^{1 / 2}} 2 \pi r \mathrm{~d} r \mathscr{P}(r, z)=\frac{64}{35} \frac{\pi r_{0}^{3} \sqrt{2}}{\alpha} \frac{\mathscr{P} \max }{P}\left(1+\frac{19}{16} \varepsilon\right)
$$

Substituting for all quantities on the RHS of (10) using the values listed in Table 1 we find that (10) gives predicted energy confinement times of $\tau_{E}=5.1 \mu$ s for the Durance et al. experiment, and $\tau_{E}=1.1 \mu \mathrm{s}$ for the Knight experiment. As shown in Table 1, Durance et al. (using numerical solutions of the Grad-Shafranov equation) explicitly quoted a measured confinement time of $5 \mu \mathrm{s}$, in close agreement with the result obtained here.

Klima (1974), by using the Maxwell stress tensor to give relationships between momentum and energy input, showed that the bulk drag torque

$$
\left\langle T_{z}\right\rangle=\int \mathrm{d}^{3} r n(\mathbf{r} \times \mathbf{F})_{z}
$$

is related to the total input power $P$ and frequency $\omega$ by

$$
\frac{\left\langle T_{z}\right\rangle}{P}=\frac{1}{\omega}
$$

The drag force is just $F=-m_{\mathrm{e}} v_{\mathrm{ei} \perp} J_{\theta} / n e$ where $-J_{\theta} / n e$ is the drift velocity associated with the current and $v_{\mathrm{ei} \perp}$ is the collision frequency associated with currents flowing perpendicular to the plasma's DC magnetic field. This perpendicular collision frequency is related to the perpendicular resistivity $\eta_{\perp}$ as $v_{\mathrm{e} \perp \perp}=n e^{2} \eta_{\perp} / m_{\mathrm{e}}$. The perpendicular value of collision frequency (which is twice the magnitude of the parallel collision frequency) is used here because in a rotamak the DC current is flowing in the toroidal direction whereas the DC magnetic field is in the poloidal direction.

The Grad-Shafranov equation describes pressure profiles and does not give any information on the separate profiles of density and temperature. Experimental data (DURANCE et al., 1987; KNIGHT, 1988) indicate that density profiles are somewhat more peaked than temperature profiles. In order to be qualitatively consistent with these observations, we will assume that the density scales radially as $n \sim \mathscr{P}^{3 / 5}$ and that the temperature scales as $T_{\mathrm{e}} \sim \mathscr{P}^{2 / 5}$. This particular (but reasonable) choice of profiles provides the substantial analytic convenience that the electron-ion collision frequency $v_{\mathrm{e} \perp \perp} \sim n / T_{\mathrm{e}}^{3 / 2}$ becomes radially uniform and so may be factored out of profile integrals. Thus (12) becomes

$$
-m_{\mathrm{e}} \frac{v_{\mathrm{ei} \perp}}{e P} \int \mathrm{d}^{3} r r J_{\theta}=\frac{1}{\omega}
$$

whereas in (10), the integration is over the plasma volume up to the separatrix. If we assume that $J_{\theta}$ is linear in $r$ as predicted by (9) then evaluation of the integral in (13) gives 


$$
J_{\theta}=-\frac{15 \alpha}{32 \sqrt{2}} \frac{e}{m_{\mathrm{e}} v_{\mathrm{ei} \perp}} \frac{P}{\pi r_{0}^{3}} \frac{r}{\omega r_{0}^{2}} .
$$

By integrating (14) up to the separatrix, the total plasma current may be found to be

$$
I=\int_{-R_{\mathrm{sep}} / \alpha}^{R_{\mathrm{sep}} / \alpha} \mathrm{d} z \int_{0}^{\left(R_{\mathrm{sep}}^{2}-\chi^{2} z^{2}\right)^{1 / 2}} J_{\phi} \mathrm{d} r=-\frac{5}{8} \frac{e}{m_{\mathrm{e}} \nu_{\mathrm{e} \perp \perp}} \frac{P}{\omega \pi r_{0}^{2}} .
$$

The collision frequency is given by

$$
v_{\mathrm{ei} \perp}=\frac{\omega_{\mathrm{pe}}^{4} Z \ln \Lambda}{3(2 \pi)^{3 / 2} n\left(\mathrm{k} T_{\mathrm{e}} / m_{\mathrm{e}}\right)^{3 / 2}}
$$

Throughout this work we will express all temperatures in electronvolts, so that the Boltzman constant is $\mathrm{k}=1.6 \times 10^{-19} \mathrm{~J} \mathrm{eV}^{-1}$. In practical units, (16a) becomes

$$
v_{\mathrm{ei} \perp}=2.9 \times 10^{-12} \frac{n Z \ln \Lambda}{T_{\mathrm{e}}^{3 / 2}} \mathrm{~s}^{-1}
$$

numerical values for the two experiments under discussion are listed in Table 1. If we use the parameters of Table 1 to evaluate the RHS of (15) we obtain predicted driven currents of magnitude $I=1.8 \mathrm{kA}$ for the Durance et al. experiment and $I=2.6 \mathrm{kA}$ for the Knight experiment, in reasonable agreement with the measured values of current listed in Table 1.

We have calculated $J_{\theta}$ in two different ways: (9) gives the current required to provide MHD pressure balance in the minor radius direction in accordance with the Solov'ev solution to the Grad--Shafranov equation, while (14) gives the current density resulting from force balance in the toroidal direction. Equating these two currents gives an expression for $\varepsilon$

$$
\varepsilon=1-\left[\frac{15 \alpha e P}{128 \omega m_{\mathrm{e}} v_{\mathrm{e} \perp \perp} \pi r_{0}^{3}}\right]^{2} \frac{\mu_{0}}{\left(1+\alpha^{2} / 4\right) \mathscr{P}_{\max }} .
$$

$\varepsilon=0$ corresponds to perfect confinement (zero pressure at the separatrix, all plasma pressure confined by poloidal field). Thus, if $\varepsilon$ is set to zero in (17) the following relation is obtained for the input power required to support a given pressure with perfect confinement:

$$
P_{\varepsilon=0}=\left[\frac{\left(1+\alpha^{2} / 4\right) \mathscr{P}_{\max }}{\mu_{0}}\right]^{1 / 2}\left[\frac{128 \omega m_{\mathrm{e}} v_{\mathrm{ei} \perp} \pi r_{0}^{3}}{15 \alpha e}\right]
$$

If the power is less than that given by (18) then $\varepsilon$ is given by 


$$
\varepsilon=1-\left[\frac{P}{P_{\delta=0}}\right]^{2} .
$$

Let us first consider how well (18) and (19) describe the Durance et al, experiment. Using the parameters of Table 1 in the RHS of (18), gives $P_{\varepsilon=0}=16.5 \mathrm{~kW}$, which when combined with the experimentally measured power of $P=17 \mathrm{~kW}$ in (19) gives a predicted $\varepsilon \approx 0$ whereas experimentally it was observed that $\varepsilon \approx 0.3$. Now let us consider how well (18) and (19) describe the Knight experiment. Here the parameters of Table 1 give $P_{\varepsilon=0}=22 \mathrm{~kW}$ whereas the injected power was $23 \pm 8 \mathrm{~kW}$. Here, again evaluation of (19) gives $\varepsilon \approx 0$ whereas experimentally it is observed that $\varepsilon \approx 0.75$. Since the right-hand side of (19) depends on the square of the ratio of two powers both of which may be subject to appreciable measurement error, and since a subtraction is involved, the discrepancy between measured and predicted values of $\varepsilon$ is not large. However, it is clear that one ought to use (18) and (19) in the following manner: make sure an experiment has input power of at least $P_{\varepsilon=0}$, but use experimental measurement to obtain the precise value of $\varepsilon$.

\section{CONFINEMENT, DENSITY, AND TEMPERATURE RELATIONS}

When the experimentally measured $\varepsilon$ is nonzero, the plasma is not being completely confined by MHD force. The particles do not vanish instantly from the device, but rather move out at the ion acoustic velocity $c_{\mathrm{s}}=\left(\gamma Z \mathrm{k} T_{\mathrm{e}} / m_{\mathrm{i}}\right)^{1 / 2}$. This worst case of confinement will be referred to here as "ion acoustic confinement".

As pointed out by DONNELly et al. (1987), when an ion-electron pair leave the plasma they not only convect their kinetic energy but also effectively remove a "latent heat" of ionization $\xi$. This latent heat energy is the bond breaking energy $(13.6 \mathrm{eV}$ for hydrogen) plus the energy per pair typically radiated by neutrals before and during ionization. According to figure 10 of HARRISON (1984) one needs an energy $\xi \approx 40$ $\mathrm{eV}$ to produce one electron-ion pair from atomic hydrogen in a plasma having Durance et al.'s parameters of $n \sim 10^{19} \mathrm{~m}^{-3}$ and $T_{\mathrm{e}} \sim 10 \mathrm{eV}$. Taking $\xi$ into account the energy confinement time $\tau_{E}$ is related to the particle confinement time by

$$
\frac{3 n \mathrm{k} T_{\mathrm{e}} / 2}{\tau_{E}}=\frac{3 n \mathrm{k} T_{\mathrm{e}} / 2+n \mathrm{k} \xi}{\tau_{\mathrm{p}}}
$$

or

$$
\tau_{E}=\frac{\tau_{\mathrm{p}}}{1+2 \xi / 3 T_{\mathrm{c}}}
$$

so that $\tau_{E} \approx \tau_{\mathrm{p}} / 4$ for the Durance et al. experiment.

The lack of confinement may not occur over the whole surface of the plasma; in general it could occur for some fraction $F$ of the surface area, where $0<F<1$. The characteristic particle confinement time then is

$$
\tau_{\mathrm{p}}=\frac{a}{F\left(\gamma Z \mathrm{k} T_{\mathrm{e}} / m_{\mathrm{i}}\right)^{1 / 2}}
$$


where $\gamma=5 / 3$ is the ratio of specific heats, $a$ is the effective distance from the magnetic axis to the wall and $T_{\mathrm{e}}$ is the temperature on the magnetic axis. Thus, the energy confinement time is

$$
\tau_{E}=\frac{a}{F\left(\gamma Z \mathrm{k} T_{\mathrm{e}} / m_{\mathrm{i}}\right)^{1 / 2}\left(1+2 \xi / 3 T_{\mathrm{e}}\right)} .
$$

In practical units this can be expressed as

$$
\tau_{E}=\frac{8.5 \times 10^{-5} a}{F\left(1+2 \xi / 3 T_{\mathrm{e}}\right)}\left(\frac{\mu}{Z T_{\mathrm{e}}}\right)^{1 / 2} \mathrm{~s}
$$

where $\mu$ is the ion mass in a.m.u. The parameters in Table 1 inserted in (22b) give ion acoustic confinement times of $0.6 / F \mu$ s for Durance et al. and $0.7 / F \mu$ s for Knight (assuming that $\xi$ is also about $40 \mathrm{eV}$ for argon). Comparison with the experimental confinement times [cf. discussion following (10)], of $5.1 \mu$ s for the Durance et al. experiment and $1.1 \mu$ s for the Knight experiment, we see that $F \approx 0.12$ for the Durance et al. experiment, whereas $F \approx 0.7$ for the Knight experiment. Thus, the energy confinement on the Durance experiment was equivalent to having acoustic-type losses from about $12 \%$ of the plasma surface, whereas the Knight experiment was equivalent to having acoustic losses from about $70 \%$ of the plasma surface. These evaluations for $F$ are consistent with the experimental observation that the Durance et al. experiment has better confinement $(\varepsilon \approx 0.3$ ) than the Knight experiment $(\varepsilon \approx 0.75$ ), but the data are too imprecise to make a more quantitative analysis.

We will now address the question of what happens when the input power is increased. One expects the temperature and the current to increase, but what happens to the density is less clear. If the plasma is not fully ionized, then increasing the power will increase the density in addition to increasing the temperature and current. However, if the plasma is fully ionized then the density cannot increase and all injected power must go into temperature increase. Of course, the amount of increase of these parameters might not be simple to calculate because $\tau_{E}$ might change as more power is injected. Since the behaviors of partially and fully ionized plasmas differ, we will discuss them separately, starting with the partially ionized case. The following analysis is presented as a plausible hypothesis which should be subject to experimental verification.

\subsection{Partially ionized plasma}

Both the Durance et al. and the Knight experiments were partially ionized. Durance et al. noted that when the input power was increased, the density went up considerably, but the temperature went up only a small amount. (This, of course, is contrary to what one would like to be the result of increased power input, namely an increase in temperature, with little or no change in density.) Durance et al. included (cf. their figure 6) a measure of the driven current as a function of input power and found that $I \sim P^{1 / 2}$. Since (15) predicts that $I \sim P / \nu_{\mathrm{ei} \perp}$, this suggests that $v_{\mathrm{e} \perp \perp} \sim P^{1 / 2}$. Thus, the collisionality is increasing with power rather than-as hoped-decreasing. Since $v_{\mathrm{ei}} \sim n / T_{\mathrm{e}}^{3 / 2}$, this is in qualitative agreement with the experimental observation that 
the temperature is nearly constant whereas the density increases when power is injected.

What we wish to show here is that it is possible to obtain a quite quantitative description of these behaviors if two plausible assumptions are made:

(i) the enegy confinement is always acoustic-like [i.e., given by (22a)],

(ii) for partially ionized plasmas the pressure profiles at different input powers are self-similar, i.e. when more power is injected, the plasma changes in such a way that $\varepsilon$ stays the same (recall $\varepsilon$ is the ratio of the pressure at the separatrix to the pressure at the magnetic axis).

We found for the Durance et al. experiment at $17 \mathrm{~kW}$ input power, that the value of $\varepsilon$ predicted by (19) was $\approx 0$. We assume then, that when the amount of injected power is varied, the plasma will change in such a way that the $\varepsilon$ predicted by (19) will always be $\approx 0$. Thus, we are assuming that the density and temperature will change such that we always have $P=P_{\varepsilon=0}$ where $P_{\varepsilon=0}$ is defined in (18). [Note that the actual measured values of $\varepsilon$ were not zero, which suggests some discrepancy in the values of power used. However, if we go to the trouble of using more accurate values of $\varepsilon$, all that will result is that $P_{\varepsilon=0}$ will be replaced by $(1-\varepsilon)^{1 / 2} P_{\varepsilon=0}$ which will not change the results by an amount exceeding the expected accuracy of the analysis. Hence, it is sensible to simply use $\varepsilon \approx 0$.]

Equation (18) predicts a scaling relation

$$
P=\lambda_{1} n^{3 / 2} T_{\mathrm{e}}^{-1}
$$

We can obtain another relation between $n, T_{\mathrm{e}}$ and $P$ if we use assumption (i) above and equate the measured confinement time (10) to the acoustic time (22a), i.e.

$$
\frac{n T_{\mathrm{e}}}{P}=\frac{\hat{\lambda}_{2}}{T_{\mathrm{e}}^{1 / 2}\left(1+\xi^{*} / T_{\mathrm{e}}\right)}
$$

where $\xi^{*}=2 \xi / 3$. (24) can be solved for $P$ in terms of $T_{\mathrm{e}}$ to obtain

$$
P=\frac{n T_{\mathrm{e}}^{3 / 2}}{\lambda_{2}}\left(1+\frac{\xi^{*}}{T_{\mathrm{e}}}\right)
$$

In the limits where $T_{\mathrm{e}} \gg \xi^{*}$ or where $T_{\mathrm{e}} \ll \xi^{*},(25 \mathrm{a})$ can be approximated as

$$
P \sim n T_{\mathrm{e}}^{1-q}
$$

where $q=-1 / 2$ if $T_{\mathrm{e}} \gg \xi^{*}$ and $q=1 / 2$ if $T_{\mathrm{e}} \ll \xi^{*}$. Equating the $P_{\mathrm{s}}$ obtained from (23) (self-similarity assumption) and from (25b) (acoustic confinement assumption) gives a relation between $n$ and $T_{e}$,

$$
T_{\mathrm{e}} \sim \frac{1}{n^{4-2 q}}
$$

If we substitute for $T_{\mathrm{e}}$ in (25b) using (26) we obtain 


$$
n \sim P^{\frac{4-2 q}{5-\frac{3 q}{3 q}}}
$$

and if we use this in (26), we obtain

$$
T_{\mathrm{e}} \sim P^{\frac{1}{5-3 q}}
$$

If we calculate the scaling of the collision frequency, we obtain

$$
v_{\mathrm{ei} \perp} \sim P^{\frac{5 / 2-2 q}{5-3 q}}
$$

The exponent of $P$ in $(29)$ has a value of $\approx \frac{1}{2}$ for $-\frac{1}{2}<q<\frac{1}{2}$. Thus, $v_{\text {ei } \perp} \sim P^{1 / 2}$ for both $T_{\mathrm{e}} \ll \xi^{*}$ and $T_{\mathrm{e}} \gg \xi^{*}$. This square root dependence of collision frequency on power is in good agreement with the experimental observations presented in figure 6 of Durance et al. and also is not very sensitive to the way $\tau_{E}$ depends on temperature.

The Durance et al. experiment is in the range where $T_{\mathrm{e}}$ is comparable to $\xi^{*}$ so that both the $q=\frac{1}{2}$ and $q=-\frac{1}{2}$ terms contribute. We now extend the above analysis to include this more complicated situation. Equating (23) to (25a) gives

$$
n=\frac{T_{\mathrm{e}}^{5}}{\left(\lambda_{1} \lambda_{2}\right)^{2}}\left(1+\frac{\xi^{*}}{T_{\mathrm{e}}}\right)^{2}
$$

or in practical units

$$
n=\frac{4.3 \times 10^{28} F^{2} T_{\mathrm{e}}^{3}\left(\xi^{*}+T_{\mathrm{e}}\right)^{2}}{a^{2}\left(\alpha^{2}+4\right)(\ln \Lambda)^{2} \mu \omega^{2} Z} \mathrm{~m}^{-3} .
$$

Inserting (30) in (25a) gives

$$
P^{*}=T^{* 7 / 2}\left(T^{*}+1\right)^{3}
$$

where $T^{*}=T_{e} / \xi^{*}$ and

$$
P^{*}=\frac{P \lambda_{1}^{2} \lambda_{2}^{3}}{\xi^{* 13 / 2}}=\frac{1.4 \times 10^{-15}\left(a / r_{0}\right)^{3} \alpha\left(\alpha^{2}+4\right)(\ln \Lambda)^{2} \mu^{3 / 2} \omega^{2} Z^{1 / 2} P}{F^{3} \xi^{* 13 / 2}} .
$$

By taking a cube root of both sides (32) can be rewritten as

$$
T^{* 2}+T^{*}-P^{* 1 / 3} / T^{* 1 / 6}=0 \text {. }
$$

We can approximate $T^{* 1 / 6} \approx 1$ in the last term for the range $0.1<T^{*}<10$, and then solve the resulting quadratic equation to obtain

$$
T^{*}=\frac{\left(1+4 P^{* 1 / 3}\right)^{1 / 2}-1}{2} .
$$


As before, this value of $T^{*}$ is used in (30) to give $n=n(P)$, and consequently the collision frequency as a function of $P$, which [as discussed following (29)] will have approximately a square root dependence on $P$.

One unexpected and surprising result of this analysis is that $P^{*}$ is independent of the size of the device, all other parameters including power kept constant, and assuming that $a / r_{0}$ is independent of the size of the device. Also, from (34) we see that $T^{*}$ is also independent of size.

We can substitute the parameters of the Durance et al. experiment in (33) to obtain $P^{*}=0.14$. Substituting this into (35) gives $T^{*}=0.38$ or $T_{\mathrm{c}}=10 \mathrm{eV}$. Inserting this value in (31) gives $n=5 \times 10^{18} \mathrm{~m}^{-3}$, the discrepancy with the actual measured value being due to the slight inconsistency in using $\varepsilon=0$ here, whereas actually $\varepsilon=0.3$. However, due to the already complicated form of these relations, and due to the uncertainty in $F$ it is not worthwhile to treat $\varepsilon$ self-consistently.

It is worth repeating the main results of this analysis : if the density is not clamped (i.e. one has a partially ionized plasma), and if the pressure profile remains self-similar when the power is varied, then both the collision frequency and the driven current scale as the square root of the input power. Additionally, the temperature will be independent of the size of the device. These results are valid even for the changes of the dependence of $\tau_{E}$ on temperature from $\tau_{E} \sim T_{\mathrm{e}}^{1 / 2}$ to $\tau_{E} \sim T_{\mathrm{e}}^{-1 / 2}$ [cf. discussion after (29)]. We also see from (31) that, when the power is increased, the density increases much faster than does $T_{\mathrm{e}}$.

\subsection{Fully ionized plasmas}

When the plasma is fully ionized, the density is clamped to be at a value corresponding to the initial filling pressure of neutral particles (assuming 100\% recycling of ions that hit the wall). Thus, (17) has the form

$$
\varepsilon=1-\lambda P^{2} T^{2} / n^{3}
$$

where $n$ is fixed and $\lambda$ is a constant. Thus, for a given density, raising $P$ decreases $\varepsilon$ until $\varepsilon$ reaches zero. Increasing power further will give more confining force than there is pressure to confine and the plasma will pinch or contract. Let us define the power threshold for which $\varepsilon=0$ to be $P_{\text {thresh }}$ since at this power the confinement becomes "perfect". There is a threshold temperature associated with the prescribed density, which is obtained by solving (31) for $T_{\mathrm{e}}$. Inserting this threshold temperature in (32) gives the threshold power. Complete ionization of the Durance et al. fill of $1.1 \mathrm{mTorr}$ would have given a density of $7 \times 10^{19} \mathrm{~m}^{-3}$. Using this density and the Table 1 values for the other parameters in (31) gives the temperature at threshold to be $20.2 \mathrm{eV}$ or $T^{*}=0.76$. Substituting this in (32) gives $P^{*}=2.1$ so from $(33)$ the required power for complete ionization would be $230 \mathrm{~kW}$.

\section{LOADING RESISTANCE}

HugRass (1985) has shown using numerical calculations that the current $I$ driven in a rotamak is given by

$$
I=\alpha_{s}(\gamma, \lambda) I_{\text {sync }}
$$


where $\gamma=\omega_{\mathrm{c} \omega} / v_{\mathrm{ei}}, \omega_{\mathrm{c} \omega}=e \tilde{B} / m_{\mathrm{e}}, \tilde{B}$ is the r.f. magnetic field strength, $\lambda=R_{\mathrm{sep}} / \delta$, $\delta=\left(2 \eta / \mu_{0} \omega\right)^{1 / 2}$ is the classical skin depth, and the resistivity $\eta=m_{\mathrm{e}} v_{\mathrm{ei}} / n e^{2}$. Also $I_{\text {sync }}$ is the current obtained when all electrons move synchronously with the rotating field, i.e. have a current density $J_{\theta}=n e \omega r$. If we integrate this synchronous current density over the cross-section of the Solov'ev profile [same integration procedure as used in (15)] then we find

$$
I_{\mathrm{sync}}=-\frac{4 \sqrt{2}}{3 \alpha} n r_{0}^{3} e \omega
$$

Hugrass's calculation (cf. figure 2 of Hugrass) for the function $\alpha_{s}(\gamma, \lambda)$ used in (37) is reproduced here as the set of dashed curves in Fig. 1.

Recall that (15) also gave an expression for the r.f.-driven current; (15) and (37) were derived using somewhat different points of view and differ nontrivially. If we equate them we obtain

$$
P=\alpha_{s}(\gamma, \lambda) \frac{32 \sqrt{2}}{15 \alpha} n m_{\mathrm{e}}\left(\omega r_{0}\right)^{2} v_{\mathrm{ei}} \pi r_{0}^{3}
$$

In a rotamak the rotating magnetic field is generated by $N$ sets of external coils which may be arranged in various configurations (e.g. one or several turns per set, near to or far from the plasma, etc.). A given coil configuration produces a certain amount of rotating magnetic field per Ampere in the coil which may be characterized as

$$
\omega_{\mathrm{c} \omega}=\beta I_{\mathrm{c}}
$$

where $I_{\mathrm{c}}$ is the amplitude of the current in the coil, and $\beta$ depends on the coil configuration. The loading of the coil sets by the plasma will be manifested by a

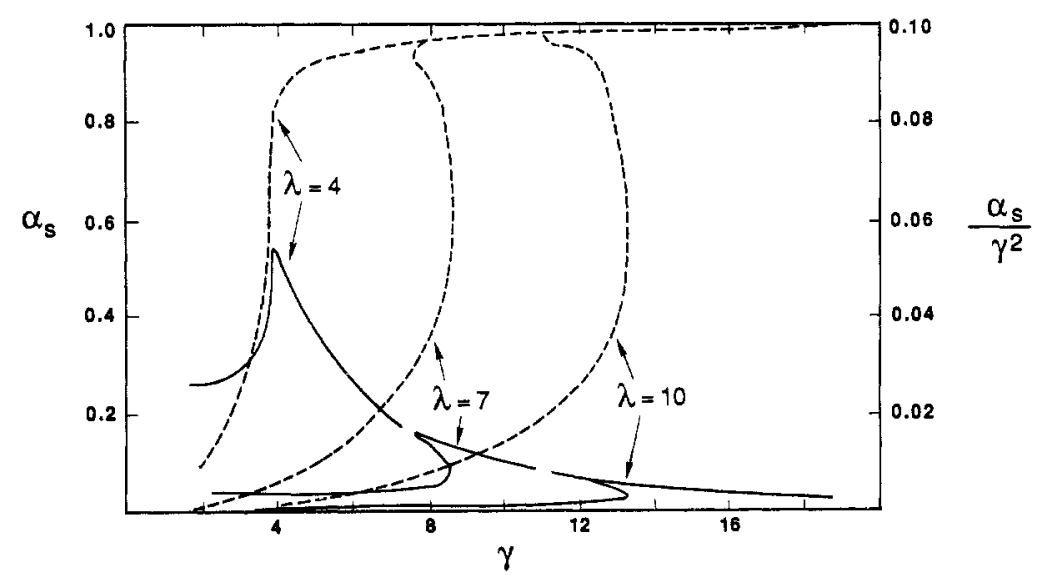

FIG. 1.-Dashed curves show HuGRass's (1985) numerical calculation of $\alpha_{s}(\gamma, \lambda)$; solid curves show $\alpha_{s} / \gamma^{2}$ vs $\gamma$, peaks of these solid curves correspond to peak in loading resistance. The slight double valued nature of the $\alpha_{s}$ curves was discussed by Hugrass; this double-valued behavior causes $\alpha_{s} / \gamma^{2}$ to become double valued as well. 
loading resistance $R_{\text {load }}$ appearing in series with each coil set, so that the power delivered to the plasma is $N I_{\mathrm{c}}^{2} R_{\text {load }} / 2$. If we replace the left-hand side of (39) by this power and use (40) to eliminate $I_{\mathrm{c}}$ in favour of $\omega_{\mathrm{c} \omega}=\gamma \nu_{\mathrm{ei}}$ we obtain

$$
R_{\text {load }}=\frac{\alpha_{s}(\gamma, \lambda)}{\gamma^{2}} \frac{\beta^{2}}{v_{\mathrm{ei}} N} \frac{64 \sqrt{2}}{15 \alpha} n m_{\mathrm{e}}\left(\omega r_{0}\right)^{2} \pi r_{0}^{3}
$$

Thus the loading resistance for a given temperature (i.e. a given $n / v_{\mathrm{ei}}$ ) depends on $\alpha_{s}(\gamma, \hat{\lambda}) / \gamma^{2}$ which is shown by the solid curves in Fig. 1 . As can be seen from Fig. 1 , $\alpha_{s} / \gamma^{2}$ peaks to a value of approximately $1 / \lambda^{2}$ when $\gamma \approx \lambda$.

In general, maximum power will be transferred to the plasma when this loading resistance is at its maximum. This is because the loading resistance appears in series with the zero-plasma r.f. coil resistance (i.e. circuit losses) and so the power that goes to the plasma is in proportion to $R_{\text {load }} /\left(R_{\text {circuitlosses }}+R_{\text {load }}\right)$. Also the loading resistance in experiments to date have been small compared to the r.f. generator impedance (1$10 \mathrm{Ohm}$ vs $10-50 \mathrm{Ohm}$ ) so that matching to the generator would be optimized by maximizing the loading resistance. Thus, the peak loading resistance (optimal operating point) is achieved by adjusting parameters such that $\gamma \approx \lambda$, or equivalently $v_{\mathrm{ei}} \approx \omega_{\mathrm{c} \omega} / \lambda$. Noting that $\lambda=R_{\text {sep }} / \delta$ and that $\delta^{2}=2\left(\eta / \mu_{0}\right) \omega=2 m_{\mathrm{e}} v_{\mathrm{ei}} / \mu_{0} \omega n e^{2}$, (41) gives the peak loading resistance to be

$$
R_{\mathrm{load}}=\frac{64 \sqrt{2}}{15}\left(\frac{m_{\mathrm{e}}}{e}\right)^{2} \frac{\pi}{\mu_{0}} \frac{r_{0}^{3} \beta^{2} \omega}{\alpha N}=4.8 \times 10^{-16} \frac{r_{0}^{3} \beta^{2} \omega}{\alpha N} \quad \text { Ohms }
$$

We note that (42) does not depend on temperature so that we can drop the proviso stated after (41) that the peak resistance was being calculated for a given temperature. Also it is interesting to note that this peak loading resistance does not depend on density either; it simply depends on geometrical factors and frequency.

It should be noted that the loading resistance predicted by (42) should be understood to be approximate, since a number of important approximations are implicit in the derivation leading to (42). Specifically: (i) Hugrass's analysis which lead to (37) assumed that the plasma was an infinitely long cylinder, whereas the analysis leading to (15) assumed that the plasma was a finite sized ellipsoid; (ii) $n$ is assumed uniform in Hugrass's analysis, whereas we have assumed that $n \sim \mathscr{P}^{3 / 5}$ [cf. discussion before (13)]; and (iii) Hugrass showed that complete penetration of the rotating field and hence $J_{\theta} \sim r$ occurs only when $\gamma \geqslant \hat{\lambda}$ whereas we are assuming that $J_{\theta} \sim r$ always (i.e. we are ignoring the skin effect that would occur when $\gamma \ll \lambda$, a reasonable assumption since we have shown that the resistance peaks when $\gamma=\hat{\lambda}$ ).

The condition $i \approx \gamma$ for optimum coupling (peak of loading resistance) can be used to obtain a relationship between density, temperature, $r_{0}$, and $\omega_{\mathrm{c} \omega}$ for which the resistance peaks, namely

$$
\nu_{\mathrm{ei}}^{1 / 2} \omega_{\mathrm{pe}} \approx \frac{c}{r_{0}} \frac{\omega_{\mathrm{c} \omega}}{\omega^{1 / 2}}
$$

or, using (16b) this gives the optimum density in practical units to be 


$$
n \approx \frac{3.1 \times 10^{12}}{r_{0}} \frac{T_{\mathrm{e}}^{3 / 4} \omega_{\mathrm{c} \omega}}{(\omega Z \ln \Lambda)^{1 / 2}} \mathrm{~m}^{-3}
$$

Let us now compare the predictions of (42) and (44) with Knight's experiment. In Knight's experiment two orthogonal coil sets were used, i.e. $N=2$. Since 120 Amperes (average of 104 Amperes in one set and 140 Amperes in the other set) in the coils produced $\approx 15 \mathrm{G}$ of rotating field, $\beta=2.2 \times 10^{6} \mathrm{~A}^{-1} \mathrm{~s}^{-1}$. Using these values of $\beta, N$ and the values of $r_{0}, \alpha$, and $\omega$ in Table 1 gives a predicted loading resistance of $R_{\text {load }}=1 \mathrm{Ohm}$ which is close to the measured load resistance of $2 P / N I_{\mathrm{c}}^{2}=1.5 \mathrm{Ohms}$. Using the Table 1 parameters for the RHS of (44) gives the optimum density for the given temperature and driving field to be $n \approx 7.3 \times 10^{18} \mathrm{~m}^{-3}$ which is close to the actual density of $1.2 \times 10^{19} \mathrm{~m}^{-3}$.

Let us also compare the predictions of (42) and (44) to the Durance et al. experiment. In this experiment again $N=2$ and the coil current was 96 Amperes (WATTERSON, 1988, private communication) giving a rotating field of $17 \mathrm{G}$ so that $\beta=3.2 \times 10^{6} \mathrm{~A}^{-1} \mathrm{~s}^{-1}$. Using these values and the Table 1 parameters gives a predicted loading resistance of $9.4 \mathrm{Ohms}$, whereas the measured loading resistance was $2 P /$ $N I_{\mathrm{c}}^{2}=1.8 \mathrm{Ohms}$. The density which peaks the loading resistance as predicted by (44) is $n=7.4 \times 10^{18} \mathrm{~m}^{-3}$. The reason for the factor of five discrepancy between the predicted and measured loading resistance is possibly related to the error resulting from simultaneously and inconsistently using Hugrass's assumption of finite cylindrical geometry for $\alpha_{s}$ in (37) with the assumption of ellipsoidal geometry for (15). This possibility seems most likely since there was much better agreement between (42) and Knight's experiment, which being very elongated more closely approximated the infinite cylinder assumed by Hugrass.

\section{EFFECT OF APPLIED DC TOROIDAL FIELD}

In our analysis we have seen that (15), the equation for current drive efficiency, does not depend on DC toroidal field. However, BERTRAM (1987) has evaluated the effect of a toroidal field on the functional dependence of $\alpha_{5}$ on $\gamma$ and has found the toroidal field makes the knee in the $\alpha_{s}$ vs $\gamma$ curve less sharp and occur at a lower value of $\gamma$. Since $\alpha_{s}(\gamma, \lambda)$ was involved in determining the plasma loading we might expect that the effect of a toroidal field is to affect the plasma loading resistance behavior.

Bertram's results (from figure 1 of his paper) for parameters of the ANSTO device and three values of toroidal field $\left(B_{\text {tor }}=0,50\right.$ and $\left.200 \mathrm{G}\right)$ are shown as dashed lines in Fig. 2. Note that Bertram's choice of $\omega=5 \times 10^{6} \mathrm{~s}^{-1}, R_{\mathrm{sep}}=0.15 \mathrm{~m}, \eta=4 \times 10^{-3}$ Ohm-m, and $n=3 \times 10^{18} \mathrm{~m}^{-3}$ correspond to $\lambda \approx 4.2$ and also define the $\gamma$ scale shown on the bottom horizontal scale of Fig. 2. These dashed lines have been scaled to evaluate $\alpha_{s} / \gamma^{2}$ which is plotted as a solid line in Fig. 2 for the three respective DC field strengths. It is clear from these plots that the effect of the toroidal field is to make the peaks of $\alpha_{s} / \gamma^{2}$ occur at a lower value of $\gamma$, i.e. at a higher collisionality. Also, it is clear that the amplitude of the peaks of $\alpha_{s} / \gamma^{2}$ are essentially independent of the strength of the toroidal field. Thus the peak loading resistance is always the same, but occurs at a higher collisionality. A given r.f. power applied to a plasma with toroidal field and with collisionality increased (so as to give the same loading resistance as a plasma 


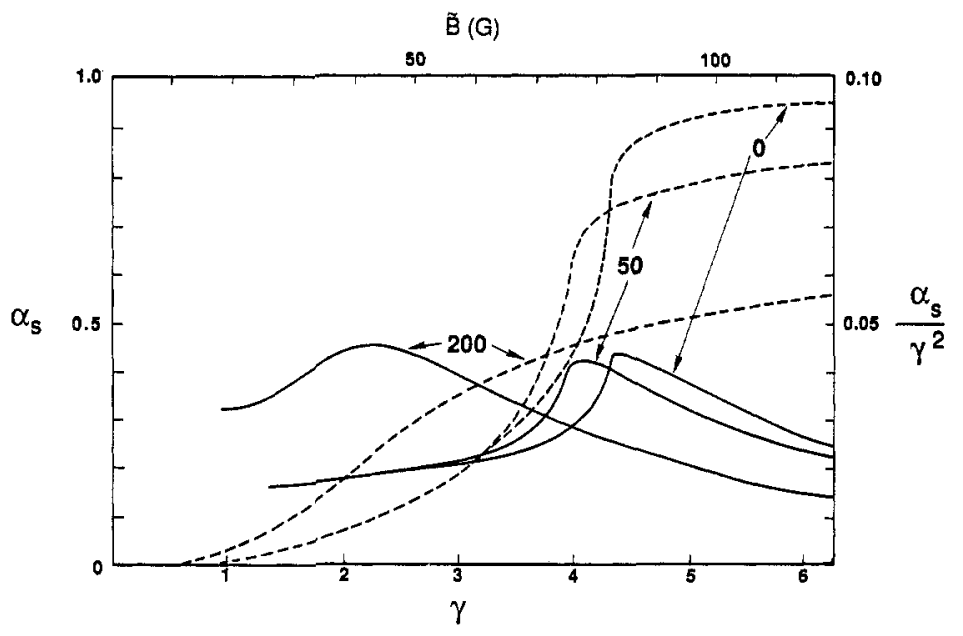

Fig. 2.-Dashed lines are from figure 1 of Bertram for applied DC toroidal field of 0,50 and $200 \mathrm{G}$. The solid curves show $\alpha_{s} / \gamma^{2}$ as scaled from the dashed curves. The effect of the DC toroidal field is to make the peaks occur at a lower value of $\gamma$, i.e. at a higher collisionality.

with no toroidal field) will give a reduced plasma current. This can be seen (i) by considering (15) which shows that the current is decreased because the collisionality is increased, or (ii) by considering Bertram's curves (dashed lines in Fig. 2) which show that $\alpha_{s}$ is reduced.

In an experiment a toroidal field may be applied without adjustment of other parameters (e.g. $\gamma$ is kept constant). It is seen from Fig. 2 that if the impedance had been matched for the case of no toroidal field, then the plasma loading resistance will drop substantially when the toroidal field is applied. This will result in poor power coupling which would also reduce the driven current.

\section{DISCUSSION}

A plausible model of the equilibrium scaling of present-day rotamaks has been given based on the assumption that the confinement is approximately acoustic for the Knight experiment and somewhat better than acoustic for the Durance $e$ al. experiment. The two main differences between the Knight and the Durance et al. experiments were (i) the Knight experiment was in argon whereas the Durance et al. experiment was in hydrogen, and (ii) the Knight plasma was highly elongated, whereas the Durance et al. plasma was nearly spherical. Thus, the difference in quality of confinement should be attributable to either (i) or (ii).

Radiation (other than the ionization-related radiation included in $\xi$ ) has not been considered in this paper so far, nor has the absolute magnitude of the energy lost by this mechanism been measured in rotamak experiments to date. Although only a careful measurement will make a definite determination of the importance of nonionization-related radiation, it appears that such radiation is either (i) not the dominant energy loss mechanism in the experiments discussed here or, less likely, (ii) if it is the dominant energy loss mechanism it is somehow occurring in a fashion that provides the energy confinement time given by (22a). In the Knight argon plasma the 
radiation could occur either from neutrals excited by electrons, or else from ions excited by electrons. In the Appendix it is argued that radiation from ions cannot be a significant loss mechanism for the Knight experiment. In the Durance et al. hydrogen experiment, line radiation could come only from electrons colliding with neutrals or from inpurity ions.

Acknowledgements-This work was supported by U.S. Department of Energy Grant DE-FG0386ER53232.

Ber TRAM W. K. (1987) J. plasma Phys. 37, 423.

\section{REFERENCES}

Collins G. A., Durance G., Hogg G. R. Tendys J. and Watterson P. A. (1988) Nucl. Fusion 28, 255.

DonNelly I. J., Rose E. K. and Cook J. L. (1987) Aust. J. Phys. 40, 393.

Durance G., Hogg G. R., Tendys J. and Watterson P. A. (1987) Plasma Physics contr. Fusion 29, 227.

Durance G., Jessup B. L., Jones I. R. and Tendys J. (1982) Phys. Rev. Lett. 48, 1252.

Harrison M. F. A. (1984) in Applied Atomic Collision Physics (edited by C. F. BARnetT and M. F. A. HaRRison), p. 395. Academic Press, New York.

Hugrass W. N. (1985) Aust. J. Phys. 38, 157.

Hugrass W. N., Jones I. R., McKenna K. F., Phillips M. G. R., Storer R. G. and Tuczek H. (1980) Phys. Rev. Lett. 44, 1679.

JoNes I. R. (1986) Comments Plasma Phys. contr. Fusion 10, 115.

Klima R. (1974) Czech J. Phys. B24, 846.

KNIGHT A. J. (1988) Ph.D. Thesis, Flinders University.

Shi X. H., Hamberger S. M. and Blackwell B. D. (1988) Experimental investigation of different configurations of a flexible heliac. Australian National University Research School of Physical Sciences report ANU-PRL PP-88/1.

Solov'Ev L. S. (1975) in Reviews of Plasma Physics (edited by M. A. LeOntovich), Vol, VI, p. 239. Consultants Bureau, New York, London.

Summers H. P. and MCWhir Ter R. W. P. (1979) Phys. B: Atom. Molec. Phys. 12, 2387.

\section{APPENDIX}

This Appendix presents two separate arguments why radiation from ions cannot be the dominant energy loss mechanism in the Knight experiment.

\section{Comparison with the Sheila heliac}

A reasonable explanation that radiation from ions must be unimportant in the Knight experiment is the following. Let us compare the plasma in the Sheila heliac at the Australian National University (SHI et al., 1988) to the Knight rotamak. Both have argon plasmas with comparable densities, temperatures and volume [the density in Sheila is $1-3 \times 10^{18} \mathrm{~m}^{-3}$, the temperature is $6-8 \mathrm{eV}$, and the volume is $10^{-3} \mathrm{~m}^{3}$; the density in the Knight experiment is $10^{19} \mathrm{~m}^{-3}$, the temperature is $6.5 \mathrm{eV}$, and the volume (up to the separatrix) is $\left.\int \mathrm{d}^{3} r=8 \pi \sqrt{2} r_{0}^{3} / 3 \alpha=1.7 \times 10^{-3} \mathrm{~m}^{3}\right]$. However, Sheila is essentially fully ionized (fill $=9 \times 10^{-5}$ Torr corresponding to an atom density of $3 \times 10^{18} \mathrm{~m}^{-3}$ ), whereas the Knight experiment is weakly ionized (fill $=8 \mathrm{mTorr}$, corresponding to an atom density of $3 \times 10^{20} \mathrm{~m}^{-3}$, corresponding to $4 \%$ ionization). Thus, radiation from Sheila would be entirely from ions, whereas for the Knight experiment the radiation would be from both ions and neutrals.

The Knight rotamak requires $22 \mathrm{~kW}$ to operate. If radiation from ions were the dominant loss mechanism then the Sheila device would also require about the same power (within a factor of two or three). However, Sheila operates with an input power of only $400 \mathrm{~W}$, which is a factor of 50 lower. Clearly, radiation loss from Sheila must be $<400 \mathrm{~W}$. Thus, the Knight rotamak cannot have radiation from ions as the dominant energy loss mechanism.

\section{Direct calculation using the Summers and McWhirter radiative power-loss functions}

SUMMERS and MCWHIRTER (1979) in their Table 7 give the radiation power-loss function for $0.5 \mathrm{eV}$ argon to be (from interpolation of the table) $\log P_{\mathrm{SM}}=-19.1$ where $P_{\mathrm{SM}}$ is in units of ergs $\mathrm{cm}^{3} \mathrm{~s}^{-1}$. Their 
power formula gives

$$
P_{\text {tadiated }}=10^{P_{S M} n^{2} V} \text { ergs s}{ }^{-1}
$$

where $n$ is the density in particles per cubic centimeter and $V$ is the volume in cubic centimeters. The ellipsoidal plasma in Knight's experiment has $V=1.7 \times 10^{3} \mathrm{~cm}^{3}$ and a peak density $n=1.2 \times 10^{13} \mathrm{~cm}^{-3}$. Using these values in the Summers-McWhirter formula gives a radiated power of $1.8 \mathrm{~kW}$. This value is an upper bound because it assumes that the entire plasma has the same density as the peak. This upper bound of radiated power is a factor of 10 lower than the input power in the Knight experiment, again indicating that radiation cannot be the dominant loss mechanism. 